\title{
The Reciprocal Theorem Method for Applied Mechanics
}

\author{
Bennosuke TANimoto*, Dr. Eng. \& Dr. Sci. \\ (Received October 10, 1960)
}

Synopsis. The bending of beams and plates is treated by the extended use of the reciprocal theorem of Maxwell and Betti. This new method eliminates simultaneous equations to be encountered in the ordinary method of analysis. In the case of plates, the use of transposed matrix is especially suited.

\section{Introductory Remarks}

The reciprocal theorem of Maxwell and Betti is well known to be a pow erful weapon in many fields of mechanics. When, for example, applied to the bending of beams, this theorem states

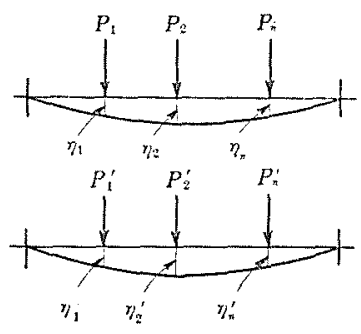

Fig. 1.
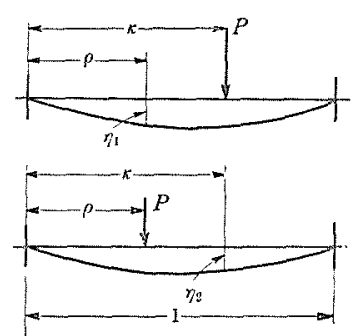

Fig. 2.
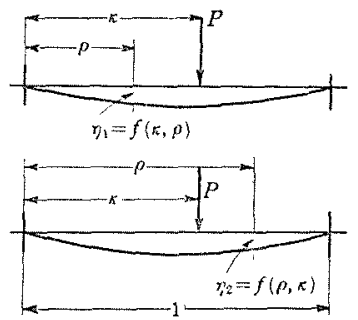

Fig. 3.

$$
\eta_{1}^{\prime} P_{1}+\eta_{2}^{\prime} P_{2}+\cdots+\eta_{n}^{\prime} P_{n}=\eta_{1} P_{1}^{\prime}+\eta_{2} P_{.}^{\prime}+\cdots+\eta_{n} P_{n}^{\prime} \text { for Fig. } 1,
$$

and accordingly

$$
\eta_{1}=\eta_{2} \text { for Fig. } 2
$$

It follows from this that,

$$
\text { if } \eta_{1}=f(\kappa, \rho) \text {, then } \eta_{2}=f(\rho, \kappa),
$$

provided that $\kappa$ and $\rho$ are respectively the same values.

The present use of the theorem is the extension of equation (3) to the

* Professor of Civil Engineering, Faculty of Engineering, Shinshu University, Nagano, Japan. 
case of Fig. 3, in which $P$ denotes a concentrated load applied at point $\rho=\kappa$, $\eta_{1}$ the deflection for the domain $0<\rho<\kappa$, and $\eta_{2}$ the deflection for the domain $\kappa<\rho<1$. If $\eta_{1}$ be denoted by $f(\kappa, \rho)$, then $\eta_{2}$ is given by interchanging $\kappa$ and $\rho$, that is by $f(\rho, \kappa)$.

In virtue of this, simultaneous equations to be encountered in the ordinary method of analysis are eliminated, and hence time and labour is much reduced. In the case of plates, the adoption of the matrix form in the course of calculation is especially suited.

\section{Bending of Beams}

A few cases of the bending of beams will be given.

The first case is the cantilever as illustrated in Fig. 4. Introducing dimensionless quantities $\rho=x / l$ and $\kappa=\xi / l$, the deflection equation for the domain $0<\rho<n$ is

$$
\frac{d^{2} \eta_{1}}{d x^{2}}=-\frac{M}{E I}=\frac{P l}{E I}(\kappa-\rho) \quad(0<\rho<\kappa),
$$

EI being flexural rigidity of the beam. Integrating this equation twice with respect to $x$, and taking $\left(\eta_{1}\right)_{\rho=0}=0$ and $\left(\frac{d \eta_{1}}{d x}\right)_{\rho=0}=0$ into account, we at once obtain

$$
\eta_{1}=\frac{P l^{3}}{6 E I}\left(3 \kappa \rho^{2}-\rho^{3}\right) \quad(0<\rho<\kappa) .
$$

Then the deflection $\eta_{2}$ for the alternative domain $\kappa<\rho<1$ is given by interchanging the letters $\kappa$ and $\rho$; that is

$$
\eta_{2}=\frac{P l^{3}}{6 E I}\left(3 \rho \kappa^{2}-\kappa^{3}\right) \quad(\kappa<\rho<1)
$$

Equations (4) and (5) are in accordance with those given in ordinary textbooks. It is to be noticed that in this case no continuity conditions at $\rho=\kappa$ are necessary.

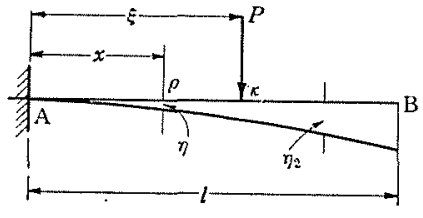

Fig. 4.

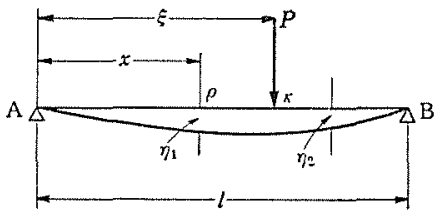

Fig. 5. 
The second example is the simple beam (Fig. 5). Deflection equation for $\eta_{1}(0<\rho<\kappa)$ becomes

$$
\frac{d^{2} \eta_{1}}{d x^{2}}=-\frac{M}{E I}=-\frac{P l}{E I}(1-\kappa) \rho \quad(0<\rho<\kappa) .
$$

Integrating this equation, with the condition $\left(\eta_{1}\right)_{0=0}=0$ at the left end $\mathrm{A}$ of the beam,

$$
\eta_{1}=-\frac{P l^{3}}{6 E I}\left[(1-\kappa) \rho^{3}+c(\kappa) \rho\right]
$$

where $c(\kappa)$ is a constant depending on the parameter $\kappa$. Then by the reciprocal theorem, the deflection $\eta_{2}(\kappa<\rho<1)$ is written

$$
\eta_{2}=-\frac{P l^{3}}{6 E I}\left[(1-\rho) \kappa^{3}+c(\rho) \kappa\right]
$$

The continuity conditions for equations (6) and (7) are

$$
\left(\eta_{1}\right)_{\rho=\kappa}=\left(\eta_{2}\right)_{\rho=\kappa}, \quad\left(\frac{d \eta_{1}}{d x}\right)_{\rho=\kappa}=\left(\frac{d \eta_{2}}{d x}\right)_{\rho=s} .
$$

The first of these equations is identically satisfied by equations (6) and (7), while the second gives

$$
\frac{d c(\kappa)}{d \kappa}-\frac{c(\kappa)}{\kappa}=3 \kappa-2 \kappa^{2}
$$

where $\left[\frac{d c(\rho)}{d \rho}\right]_{\rho=x}=\frac{d c(\kappa)}{d \kappa}$, since $c(\kappa)$ in equation (6) is independent of $\rho$. The general solution of the differential equation above can easily be found to be

$$
c(\kappa)=A \kappa-(2-\kappa)(1-\kappa) \kappa,
$$

$A$ being a mere constant. Substituting this solution into equation (7), and considering the condition $\left(\eta_{2}\right)_{\rho=1}=0$ at the right end $\mathrm{B}$, we obtain $A=0$.

Hence the wanted deflections $\eta_{1}$ and $\eta_{2}$ become respectively

$$
\left.\begin{array}{ll}
\eta_{1}=\frac{P l^{3}}{6 E I}(1-\kappa)\left[(2-\kappa) \kappa-\rho^{2}\right] \rho & (0<\rho<\kappa), \\
\eta_{2}=\frac{P l^{3}}{6 E I}(1-\rho)\left[(2-\rho) \rho-\kappa^{2}\right] \kappa & (\kappa<\rho<1)
\end{array}\right\}
$$


This result is in accordance with that given in ordinary textbooks.

The third example is the beam indicated in Fig. 6. This beam is known to be a statically indeterminate beam of one degree. The reaction $R_{\mathrm{A}}$ at the left end A may easily be found by using the ordinary reciprocal theorem, equation (1), from which we have

$$
R_{\mathrm{A}}=\frac{P}{2}(1-\pi)^{2}(2+\kappa) .
$$

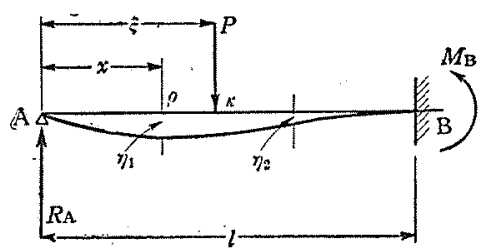

Fig. 6.

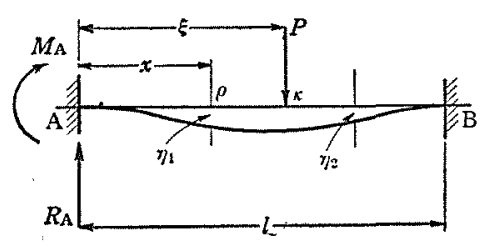

Fig. 7.

Then the deflection equation becomes

$$
\frac{d^{2} \eta_{1}}{d x^{2}}=-\frac{M}{E I}=-\frac{P l}{2 E I}(1-\kappa)^{2}(2+\kappa) \rho \quad(0<\rho<\kappa) .
$$

Integrating this, with the condition $\left(\eta_{1}\right)_{\rho=0}=0$, we have

$$
\eta_{1}=-\frac{P l^{3}}{12 E I}\left[(1-\kappa)^{2}(2+\kappa) \rho^{3}+c(\kappa) \rho\right] \quad(0<\rho<\kappa),
$$

$c(\kappa)$ being constant. Then by the reciprocal theorem

$$
\eta_{2}=-\frac{P l^{3}}{12 E I}\left[(1-\rho)^{2}(2+\rho) \kappa^{3}+c(\rho) \kappa\right] \quad(\kappa<\rho<1) .
$$

The continuity condition at point $\rho=\kappa$, equations (8), affords

$$
\frac{d c(\kappa)}{d \kappa}-\frac{c(\kappa)}{\kappa}=6 \kappa(1-\kappa)
$$

the general solution of which is

$$
c(\kappa)=A \kappa+3 \kappa^{2}(2-\kappa) .
$$

The conditions at the right end B are $\left(\eta_{2}\right)_{\rho=1}=0$ and $\left(\frac{d \eta_{2}}{d x}\right)_{\rho=1}=0$, which afford $A=-3$.

Hence the wanted deflections $\eta_{1}$ and $\eta_{2}$ become 


$$
\left.\begin{array}{ll}
\eta_{1}=\frac{P l^{3}}{12 E I}(1-\kappa)^{2}\left[3 \kappa \rho-(2+\kappa) \rho^{3}\right] & (0<\rho<\kappa), \\
\eta_{2}=\frac{P l^{3}}{12 E I}(1-\rho)^{2}\left[3 \rho \kappa-(2+\rho) \kappa^{3}\right] & (\kappa<\rho<1)
\end{array}\right\} \quad \text { (Fig. 6). }
$$

This result is in accordance with that given in ordinary textbooks.

The fourth example is the beam indicated in Fig. 7. By the ordinary recinrocal theorem or otherwise, we have

$$
R_{\mathrm{A}}=P(1-\kappa)^{2}(1+2 \kappa), \quad M_{\mathrm{A}}=-P l \kappa(1-\kappa)^{2} .
$$

The deflection equation then becomes

$$
\frac{d^{2} \eta_{1}}{d x^{2}}=-\frac{M}{E I}=\frac{P l}{E I}(1-\kappa)^{2}[\kappa-(1+2 \kappa) \rho] \quad(0<\rho<\kappa) .
$$

Integrating this, with the condition that the left end $A$ is clamped, we simply have

$$
\eta_{1}=\frac{P l^{3}}{6 E I}(1-\kappa)^{2}\left[3 \kappa \rho^{2}-(1+2 \kappa) \rho^{3}\right] \quad(0<\rho<\kappa, \text { Fig. } 7),
$$

so that the deflection of the alternative domain is

$$
\eta_{2}=\frac{P l^{3}}{6 E I}(1-\rho)^{2}\left[3 \rho \kappa^{2}-(1+2 \rho) \kappa^{3}\right] \quad(\kappa<\rho<1, \text { Fig. } 7) .
$$

The result obtained is in accordance with that given in ordinary textbooks.

The last example is the two-spanned continuous beam, in which for convenience equal span is assumed, as given in Fig. 8. By the ordinary reciprocal

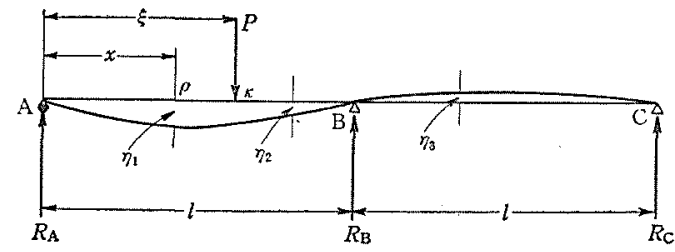

Fig. 8.

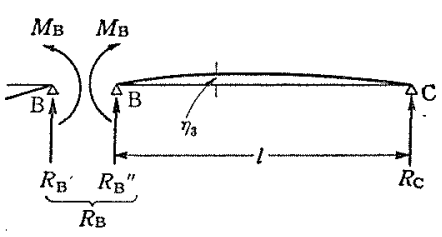

Fig. 9.

theorem, we first have

$$
R_{\mathrm{A}}=\frac{P}{4}\left(4-5 \kappa+\kappa^{3}\right), \quad R_{\mathrm{B}}=\frac{P}{2} \kappa\left(3-\kappa^{2}\right), \quad R_{\mathrm{C}}=-\frac{P}{4} \kappa\left(1-\kappa^{2}\right) .
$$


The deflection equation for $\eta_{1}$ becomes

$$
\frac{d^{2} \eta_{1}}{d x^{2}}=-\frac{M}{E I}=-\frac{P l}{4 E I}\left(4-5 \kappa+\kappa^{3}\right) \rho \quad(0<\rho<\kappa) .
$$

Integrating this, with the condition $\left(\eta_{1}\right)_{\rho=0}=0$, we have

$$
\eta_{1}=-\frac{P l^{3}}{24 E I}\left[\left(4-5 \kappa+\kappa^{3}\right) \rho^{3}+c(\kappa) \rho\right] \quad(0<\rho<\kappa) ;
$$

and accordingly

$$
\eta_{2}=-\frac{P l^{3}}{24 E I}\left[\left(4-5 \rho+\rho^{3}\right) \kappa^{3}+c(\rho) \kappa\right] \quad(\kappa<\rho<1\rangle .
$$

The continuity condition at $\rho=\kappa$ affords

$$
\frac{d c(\kappa)}{d \kappa}-\frac{c(\kappa)}{\kappa}=2 \kappa(6-5 \kappa),
$$

the general solution of which is

$$
c(\kappa)=A \kappa+(12-5 \kappa) \kappa^{2} .
$$

The condition $\left(\eta_{2}\right)_{0=1}=0$ at the intermediate support B gives $A=-7$. Hence we obtain

$$
\left.\begin{array}{ll}
\eta_{1}=\frac{P l^{3}}{24 E I}(1-\kappa)\left[(7-5 \kappa) \kappa \rho-\left(4-\kappa-\kappa^{2}\right) \rho^{3}\right] & (0<\rho<\kappa), \\
\eta_{2}=\frac{P l^{3}}{24 E I}(1-\rho)\left[(7-5 \rho) \rho \kappa-\left(4-\rho-\rho^{2}\right) \kappa^{3}\right] & (\kappa<\rho<1)
\end{array}\right\}
$$

Our last step is to find $\eta_{3}$. To do this we first have (Fig. 9)

$$
\begin{aligned}
& M_{\mathrm{B}}=-E I\left(\frac{d^{2} \eta_{\mathrm{2}}}{d x^{2}}\right)_{\rho=1}=-\frac{P l}{4} \kappa\left(1-\kappa^{2}\right), \\
& R_{\mathrm{B}}^{\prime}=-E I\left(\frac{d^{3} \eta_{\mathrm{\rho}}}{d x^{3}}\right)_{\rho=1}=\frac{P}{4} \kappa\left(5-\kappa^{2}\right),
\end{aligned}
$$

and

$R_{\mathrm{B}}^{\prime}$ being a part of $R_{\mathrm{B}}$, which plays as the reaction as if $\mathrm{AB}$ were a singlespanned beam. Then the remaining part of $R_{\mathrm{B}}$, say $R_{\mathrm{B}}^{\prime \prime}$, which is $R_{\mathrm{B}}^{\prime \prime}=R_{\mathrm{B}}$ $-R_{\mathrm{B}}^{\prime}$, is given by

$$
R_{\mathrm{B}}^{\prime \prime}=R_{\mathrm{B}}-R_{\mathrm{B}}^{\prime}=\frac{P}{2} \kappa\left(3-\kappa^{2}\right)-\frac{P}{4} \kappa\left(5-\kappa^{2}\right)=\frac{P}{4} \kappa\left(1-\kappa^{2}\right) .
$$

Hence the deflection equation for $\eta_{3}$ becomes 


$$
\frac{d^{2} \eta_{\mathrm{B}}}{d x^{2}}=-\frac{M}{E I}=-\frac{1}{E I}\left(M_{\mathrm{B}}+R_{\mathrm{B}}^{\prime \prime} x\right)=\frac{P l}{4 E I} \kappa\left(1-\kappa^{2}\right)(1-\rho)
$$

or, on integration with the conditions $\left(\eta_{3}\right)_{\rho}=0=0$ and $\left(\eta_{3}\right)_{\rho=1}=0$, we obtain

$$
r_{3}=-\frac{P l^{3}}{24 E I} \kappa\left(1-\kappa^{2}\right)\left(2 \rho-3 \rho^{2}+\rho^{3}\right) \quad \text { (Fig. 8), }
$$

$\rho$ being measured from the intermediate support $\mathrm{B}$ to the right.

From the above analyses, it can be seen that the method here presented is a sort of "the method of initial conditions", provided that statically-indeterminate quantities are known. The present method might be applicable to the analysis of the rahmen and the arch structures.

However, when the system becomes complicated and its order of indeterminateness is very high, the above method would necessitate some modification, and then recourse would be given by such as the Southwell's relaxation method. In fact, simple cases as above may be treated by not assuming stat. ically-indeterminate reactions and moments, the method of which is similar to that given in the subsquent article. Apart from this line of investigation, we shall proceed on the bending of plates.

\section{Bending of Plates}

The first example of the present method applied to plate problems is the case in which a rectangular plate is subjected to an eccentric concentrated load, the four sides of which are supposed to be of simple support. Rectangular coordinates are taken as shown in Fig. 10, in which both sides are denoted by $a$ and $b$, and the concentrated load $P$ is applied at point $A(\xi, \eta)$.

The deflection $w$ of plate in general satisfies the biharmonic equation

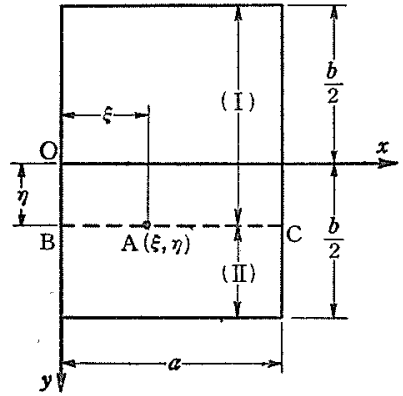

Fig. 10 .

$$
\nabla^{4} w=\left(\frac{\partial^{2}}{\partial x^{2}}+\frac{\partial^{2}}{\partial y^{2}}\right)^{2} w=0
$$

in the domain where there are no loads applied. If the plate under considera- 
tion be divided into two parts (I) and (II) by the straight line $\mathrm{BC}(y=\eta)$ parallel to the axis of $\mathrm{O} x$, the deflection $w_{\mathrm{I}}$ for the first part $(\mathrm{I}) \quad\left(-\frac{1}{2} b<y<\eta\right)$ takes the form

$$
w_{I}=\sum_{n=1}^{\infty}[A(\kappa) \operatorname{ch} \rho+B(\kappa) \rho \operatorname{sh} \rho+C(\kappa) \operatorname{sh} \rho+D(\kappa) \rho \operatorname{ch} \rho] \sin \frac{n \pi x}{a},
$$

in which for brevity

$$
\rho=\frac{n \pi y}{a}, \quad \kappa=\frac{n \pi \eta}{a}
$$

$A(\kappa), B(\kappa), C(\kappa), D(\kappa)$ being constants depending on the parameter $\kappa$. Then by the reciprocal theorem the deflection $w_{\text {II }}$ for the second part (II) $\left.\quad \eta<y<\frac{1}{2} b\right)$ is given by interchanging $\rho$ and $\pi$ in equation (16); that is

$$
w_{\mathrm{II}}=\sum_{n=1}^{\infty}[A(\rho) \operatorname{ch} \kappa+B(\rho) \kappa \operatorname{sh} \kappa+C(\rho) \operatorname{sh} \kappa+D(\rho) \kappa \operatorname{ch} \kappa] \sin \frac{n \pi x}{a} .
$$

Equations (16) and (17) satisfy the condition that the sides $x=0$ and $x=a$ are of simple support.

Now since the function (17) must satisfy the biharmonic equation (15), $A(\kappa), \cdots, D(\kappa)$ take the form

$$
\left(\begin{array}{l}
A(\kappa) \\
B(\kappa) \\
C(\kappa) \\
D(\kappa)
\end{array}\right)=\left(\begin{array}{llll}
a_{1} & a_{2} & a_{3} & a_{4} \\
b_{1} & b_{2} & b_{3} & b_{4} \\
c_{1} & c_{2} & c_{3} & c_{4} \\
d_{1} & d_{2} & d_{3} & d_{4}
\end{array}\right)\left(\begin{array}{c}
\operatorname{ch} \kappa \\
\kappa \operatorname{sh} \kappa \\
\operatorname{sh} \kappa \\
\kappa \operatorname{ch} \kappa
\end{array}\right)
$$

where $a_{1}, a_{2}, \cdots, d_{4}$ are independent of $\kappa$. In what follows matrix form will be used exclusively, which greatly facilitates our calculations. Then by substitution equations (16) and (17) are written in the forms

$$
\begin{aligned}
& w_{\mathrm{I}}=\sum_{n=1}^{\infty}[\operatorname{ch} \rho, \rho \operatorname{sh} \rho, \operatorname{sh} \rho, \rho \operatorname{ch} \rho]\left[\begin{array}{llll}
a_{1} & a_{2} & a_{3} & a_{4} \\
b_{1} & b_{2} & b_{3} & b_{4} \\
c_{1} & c_{2} & c_{3} & c_{4} \\
d_{1} & d_{2} & d_{3} & d_{4}
\end{array}\right]\left[\begin{array}{c}
\operatorname{ch} \kappa \\
\kappa \operatorname{sh} \kappa \\
\operatorname{sh} \kappa \\
\kappa \operatorname{ch} \kappa
\end{array}\right) \sin \frac{n \pi x}{a} \\
& w_{\mathrm{II}}=\sum_{n=1}^{\infty}[\operatorname{ch} \kappa, \kappa \operatorname{sh} \kappa, \operatorname{sh} \kappa, \kappa \operatorname{ch} \kappa]\left[\begin{array}{llll}
a_{1} & a_{2} & a_{3} & a_{4} \\
b_{1} & b_{2} & b_{3} & b_{4} \\
c_{1} & c_{2} & c_{3} & c_{4} \\
d_{1} & d_{2} & d_{3} & d_{4}
\end{array}\right]\left[\begin{array}{c}
\operatorname{ch} \rho \\
\rho \operatorname{sh} \rho \\
\operatorname{sh} \rho \\
\rho \operatorname{ch} \rho
\end{array}\right] \sin \frac{n \pi x}{a}
\end{aligned}
$$


and the latter can be rearranged in the form

$$
w_{11}=\sum_{n=1}^{\infty}[\operatorname{ch} \rho, \rho \operatorname{sh} \rho, \operatorname{sh} \rho, \rho \operatorname{ch} \rho]\left[\begin{array}{llll}
a_{1} & b_{1} & c_{1} & d_{1} \\
a_{2} & b_{2} & c_{2} & d_{2} \\
a_{3} & b_{3} & c_{3} & d_{3} \\
a_{4} & b_{4} & c_{4} & d_{4}
\end{array}\right]\left[\begin{array}{c}
\operatorname{ch} \kappa \\
\kappa \operatorname{sh} \kappa \\
\operatorname{sh} \kappa \\
\kappa \operatorname{ch} \kappa
\end{array}\right) \sin \frac{n \pi x}{a} .
$$

The coefficient matrices consisting of $a_{1}, a_{2}, \cdots, d_{4}$ in equations (19) and (21) are of transposed matrix each other, and it can be stated that the reciprocity in equations (16) and (17) is the same thing as the transposableness of the coefficient matrices.

The problem to be solved then reduces to determine the coefficient matrices so as to satisfy the continuity conditions along the straight line $\mathrm{BC}$ where $y=\eta$ and the supporting conditions along the sides $y= \pm \frac{b}{2}$. These conditions will be taken into account successively.

The continuity conditions are the continuity of deflections, slopes and bending moments of the domains (I) and (II) along the straight line $y=\eta$, that is $\rho=\kappa$, and as to the shearing forces there must be a discontinuity caused by the point load $P$, which may be represented in the form of Fourier sine series. Thus they take in this case the expressions

$$
\left.\begin{array}{l}
w_{\mathrm{I}}=w_{\mathrm{II}}, \quad \frac{\partial w_{\mathrm{I}}}{\partial \rho}=\frac{\partial w_{\mathrm{II}}}{\partial \rho}, \quad \frac{\partial^{2} w_{\mathrm{I}}}{\partial \rho^{2}}=\frac{\partial^{2} w_{\mathrm{II}}}{\partial \rho^{2}}, \\
\frac{\partial^{3} w_{\mathrm{I}}}{\partial \rho^{3}}-\frac{\partial^{3} w_{\mathrm{II}}}{\partial \rho^{3}}=-\frac{2 P a^{2}}{\pi^{3} D} \sum_{n=1}^{\infty} \frac{1}{n^{3}} \sin \frac{n \pi \xi}{a} \sin \frac{n \pi x}{a}
\end{array}\right\}(\rho=\kappa),
$$

where $D$ is the flexural rigidity of the plate, i.e. $D=E h^{3} / 12\left(1-\nu^{2}\right)$. The first of equations (22) is identically satisfied in virtue of the forms of equations (19) and (20). The second to the fourth in equations (22) will be considered. To do this it will be convenient to refer to the successive derivatives

$$
\left[\begin{array}{c}
1 \\
\frac{\partial}{\partial \rho} \\
\frac{\partial^{2}}{\partial \rho^{2}} \\
\frac{\partial^{3}}{\partial \rho^{3}}
\end{array}\right]\left[\begin{array}{llll}
e_{1} & e_{2} & e_{3} & e_{4}
\end{array}\right]\left[\begin{array}{c}
\operatorname{ch} \rho \\
\rho \operatorname{sh} \rho \\
\operatorname{sh} \rho \\
\rho \operatorname{ch} \rho
\end{array}\right]=\left[\begin{array}{cccc}
e_{1} & e_{2} & e_{3} & e_{4} \\
e_{3}+e_{4} & e_{4} & e_{1}+e_{2} & e_{2} \\
e_{1}+2 e_{2} & e_{2} & e_{3}+2 e_{4} & e_{4} \\
e_{3}+3 e_{4} & e_{4} & e_{1}+3 e_{2:} & e_{2}
\end{array}\right]\left(\begin{array}{l}
\operatorname{ch} \rho \\
\rho \operatorname{sh} \rho \\
\operatorname{sh} \rho \\
\rho \operatorname{ch} \rho
\end{array}\right)
$$

where $e$ represents one of $a, b, c, d$. Then after a little rearrangement the 
continuity conditions (22) afford

$$
\left(\begin{array}{cccc}
a_{1} & a_{2} & a_{3} & a_{4} \\
b_{1} & b_{2} & b_{3} & b_{4} \\
c_{1} & c_{2} & c_{3} & c_{4} \\
d_{1} & d_{2} & d_{3} & d_{4}
\end{array}\right)=\left(\begin{array}{cccc}
a_{1} & a_{2} & a_{3} & a_{4} \\
a_{2} & b_{2} & b_{3} & b_{4} \\
a_{3}+\frac{\alpha}{2} & b_{3}-\frac{\alpha}{2} & c_{3} & c_{4} \\
a_{4}-\frac{\alpha}{2} & b_{4} & c_{4} & d_{4}
\end{array}\right)
$$

where for brevity

$$
\alpha=\frac{2 P a^{2}}{\pi^{3} D} \cdot \frac{1}{n^{3}} \sin \frac{n \pi \xi}{a}
$$

It is noted here that, as to the last of equations (22), reference should be made to the identity $\operatorname{ch}^{2} \kappa-\operatorname{sh}^{2} \kappa=1$.

The remaining conditions are then those along the sides $y=\mp \frac{b}{2}$. Assuming these sides to be of simple support, we have

$$
\begin{array}{ll}
\left(w_{\mathrm{I}}\right)_{\rho=-\lambda}=0, & \left(\frac{\partial^{2} w_{\mathrm{I}}}{\partial \rho^{2}}\right)_{\rho=-\lambda}=0, \\
\left(w_{\mathrm{II}}\right)_{\rho=\lambda}=0, & \left(\frac{\partial^{2} w_{\mathrm{II}}}{\partial \rho^{2}}\right)_{\rho=\lambda}=0,
\end{array}
$$

where

$$
\lambda=\frac{n \pi b}{2 a}
$$

The formers of these equations afford respectively

$$
\left[\begin{array}{llll}
a_{1} & b_{1} & c_{1} & d_{1} \\
a_{2} & b_{2} & c_{2} & d_{2} \\
a_{3} & b_{3} & c_{3} & d_{3} \\
a_{4} & b_{4} & c_{4} & d_{4}
\end{array}\right]\left[\begin{array}{c}
\operatorname{ch} \lambda \\
\lambda \operatorname{sh} \lambda \\
-\operatorname{sh} \lambda \\
-\lambda \operatorname{ch} \lambda
\end{array}\right]=0, \quad\left[\begin{array}{llll}
a_{1} & a_{2} & a_{3} & a_{4} \\
b_{1} & b_{2} & b_{3} & b_{4} \\
c_{1} & c_{2} & c_{3} & c_{4} \\
d_{1} & d_{2} & d_{3} & d_{4}
\end{array}\right]\left[\begin{array}{c}
\operatorname{ch} \lambda \\
\lambda \operatorname{sh} \lambda \\
\operatorname{sh} \lambda \\
\lambda \operatorname{ch} \lambda
\end{array}\right]=0 .
$$

As to the latters of equations (26) and (27), it is preferable, instead of the forms as they stand, to write

$$
\left(\frac{\partial^{2} w_{\mathrm{I}}}{\partial \rho^{2}}-w_{\mathrm{I}}\right)_{\rho=-\lambda}=0, \quad\left(\frac{\partial^{2} w_{\mathrm{II}}}{\partial \rho^{2}}-w_{\mathrm{II}}\right)_{\rho=\lambda}=0 .
$$

Referring to the derivatives (23), equations (29) afford 


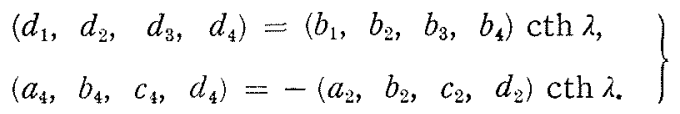

Thus all the coefficients $a_{1}, a_{2}, \cdots, d_{4}$ can be determined by equations (24), (28) and (30). First, substituting equations (30) into equations (24), we have

$$
\left[\begin{array}{cccc}
a_{1} & a_{2} & a_{3} & a_{4} \\
b_{1} & b_{2} & b_{3} & b_{4} \\
c_{1} & c_{2} & c_{3} & c_{4} \\
d_{1} & d_{2} & d_{3} & d_{4}
\end{array}\right]=\left[\begin{array}{cccc}
a_{1} & -\frac{\alpha}{4} \operatorname{th} \lambda & a_{3} & \frac{\alpha}{4} \\
-\frac{\alpha}{4} \operatorname{th} \lambda & 0 & \frac{\alpha}{4} & 0 \\
a_{3}+\frac{\alpha}{2} & -\frac{\alpha}{4} & c_{3} & \frac{\alpha}{4} \operatorname{cth} \lambda \\
-\frac{\alpha}{4} & 0 & \frac{\alpha}{4} \operatorname{cth} \lambda & 0
\end{array}\right]
$$

Equations (28), consisting of eight equations, must be satisfied simultaneously by a set of certain values of $a_{1}, a_{2}, \cdots, d_{4}$. We see at once that the second, fourth, sixth and eighth in equations (28) are identically satisfied, while the remaining four equations, with the substitution of equations (31), afford

$$
\begin{aligned}
& a_{1}-a_{3} \operatorname{th} \lambda=-\frac{\alpha}{4}\left(\lambda-2 \operatorname{th} \lambda-\lambda \operatorname{th}^{2} \lambda\right), \\
& a_{3}-c_{3} \operatorname{th} \lambda=-\frac{\alpha}{4} \lambda(\operatorname{th} \lambda-\operatorname{cth} \lambda), \\
& a_{1}+a_{3} \operatorname{th} \lambda=-\frac{\alpha}{4} \lambda\left(1-\operatorname{th}^{2} \lambda\right), \\
& a_{3}+c_{3} \operatorname{th} \lambda=-\frac{\alpha}{4}(2-\lambda \operatorname{th} \lambda+\lambda \operatorname{cth} \lambda) .
\end{aligned}
$$

The first and the third of these equations afford the values of coefficients $a_{1}$ and $c_{3}$, while the second and the fourth afford those of $a_{3}$ and $c_{3}$. Thus we obtain

$$
\begin{aligned}
& a_{1}=-\frac{\alpha}{4}\left(\lambda-\operatorname{th} \lambda-\lambda \operatorname{th}^{2} \lambda\right) \\
& a_{3}=-\frac{\alpha}{4} \\
& c_{3}=\frac{\alpha}{4}\left(\lambda-\operatorname{cth} \lambda-\lambda \operatorname{cth}^{2} \lambda\right)
\end{aligned}
$$

It is to be noted that in this case the same value of $a_{3}$ has resulted from the dif ferent sets of equations, and this would sometimes provide a practical - but 
important - means for checking that the whole system treated is a possible one on the standpoint of mechanics, and that the preceding calculations are correct.

The remaining coefficients $a_{2}, a_{4}$, etc. can at once be determined by substitituting equations (32) into equations (31). In this way the coefficient matrix in equation (19) has completely been determined. Hence the deflection $w_{1}$ for the domain $-1 b<y<\eta$ becomes

$$
\begin{aligned}
& \cdot v=\sum_{n=1}^{\infty}[\operatorname{ch} \rho, \rho \operatorname{sh} \rho, \operatorname{sh} \rho, \rho \operatorname{ch} \rho] \\
& \times \frac{\alpha}{4}\left(\begin{array}{cccc}
-\lambda+\operatorname{th} \lambda+\lambda \operatorname{th}^{2} \lambda, & -\operatorname{th} \lambda, & -1, & 1 \\
-\operatorname{th} \lambda, & 0, & 1, & 0 \\
1, & -1, & \lambda-\operatorname{cth} \lambda-\lambda \operatorname{cth}^{2} \lambda, & \operatorname{cth} \lambda \\
-1, & 0, & \operatorname{cth} \lambda, & 0
\end{array}\right]\left(\begin{array}{c}
\operatorname{ch} \kappa \\
\pi \operatorname{sh} \kappa \\
\operatorname{sh} \kappa \\
\kappa \operatorname{ch} \kappa
\end{array}\right) \sin \frac{n \pi x}{a}
\end{aligned}
$$

and accordingly, by transposing the coefficient matrix, the deflection $w_{\mathrm{II}}$ for the domain $\eta<y<3 b$ becomes

$$
\begin{aligned}
& w_{I I}=\sum_{n=1}^{\infty}[\operatorname{ch} \rho, \rho \operatorname{sh} \rho, \operatorname{sh} \rho, \rho \operatorname{ch} \rho] \\
& \times \frac{\alpha}{4}\left[\begin{array}{cccc}
-\lambda+\operatorname{th} \lambda+\lambda \operatorname{th}^{2} \lambda, & -\operatorname{th} \lambda, & 1, & -1 \\
-\operatorname{th} \lambda, & 0, & -1, & 0 \\
-1, & 1, & \lambda-\operatorname{cth} \lambda-\lambda \operatorname{cth}^{2} \lambda, & \operatorname{cth} \lambda \\
1, & 0, & \operatorname{cth} \lambda, & 0
\end{array}\right]\left[\begin{array}{c}
\operatorname{ch} \kappa \\
\kappa \operatorname{sh} \kappa \\
\operatorname{sh} \kappa \\
\kappa \operatorname{ch} \kappa
\end{array}\right] \sin \frac{n \pi \pi x}{a}
\end{aligned}
$$

where, as before,

$$
\alpha=\frac{2 P a^{2}}{\pi^{3} D} \cdot \frac{1}{n^{3}} \sin \frac{n \pi \xi}{a}, \quad \lambda=\frac{n \pi b}{2 a}, \quad \kappa=\frac{n \pi \eta}{a}, \quad \rho=\frac{n \pi y}{a} .
$$

We see that the solution obtained in equations (33) and (34) is in accordance with that obtained by the ordinary method of analysis, and that time and labour is much reduced by the present method. Numerical evaluation of the solution may also be effected efficiently from the above forms of solution.

As a simple extension of the above solution, we take a two-spanned continuous plate subjected to a concentrated load $P$. Reference is again made to Fig. 10, in which the axis of $O x$ is supposed to be the supporting line, all the other conditions being assumed to be the same as those of the single-spanned 
plate above. Let concentrated loads $P q(\xi) d \xi$ be distributed along the axis of $\mathrm{O} x, q(\xi)$ being a distribution function. The deflection $w_{\mathrm{II}}^{\prime}$ in the domain $0<$ $y<\frac{1}{3} b$ due to the distributed load is obtained by putting $\kappa=0$ in equation (34), and by integrating the result from 0 to $a$; that is

$$
\begin{aligned}
w_{\mathrm{II}}^{1}=\frac{P a^{2}}{2 \pi^{3} D} & \sum_{n=1}^{\infty} \frac{1}{n^{3}} \int_{0}^{a} q(\xi) \sin \frac{n \pi \xi}{a} d \xi \cdot[\operatorname{ch} \rho, \rho \operatorname{sh} \rho, \operatorname{sh} \rho, \rho \operatorname{ch} \rho] \\
& \times \operatorname{col}\left(-\lambda+\operatorname{th} \lambda+\lambda \operatorname{th}^{2} \lambda,-\operatorname{th} \lambda,-1,1\right) \sin \frac{n \pi x}{a},
\end{aligned}
$$

where, for saving space, the parentheses col ( ) denote a column vector.

On the other hand, when a concentrated load $P$ is applied at point $\mathrm{A}(\xi, \eta)$ the deflection $w_{\mathrm{I}}$ in the domain $-\frac{1}{2} b<y<\eta$ is given by equation (33), which for $y=0$ takes the value

$$
\begin{aligned}
\left(w_{\mathrm{I}}\right)_{y=0}= & \frac{P a^{2}}{2 \pi^{3} D} \sum_{n=1}^{\infty} \frac{1}{n^{3}} \sin \frac{n \pi \xi}{a}[\operatorname{ch} \kappa, \kappa \operatorname{sh} \kappa, \operatorname{sh} \kappa, \kappa \operatorname{ch} \kappa] \\
& \times \operatorname{col}\left(-\lambda+\operatorname{th} \lambda+\lambda \operatorname{th}^{2} \lambda,-\operatorname{th} \lambda,-1,1\right) \sin \frac{n \pi x}{a} .
\end{aligned}
$$

Since the deflection along the axis of $O x$ must vanish, we have

from which

$$
\left(w_{\mathrm{II}}^{\prime}\right)_{y=0}+\left(w_{1}\right)_{y=0}=0,
$$

$$
\begin{aligned}
(\lambda- & \left.\operatorname{th} \lambda-\lambda \operatorname{th}^{2} \lambda\right) \int_{0}^{a} q(\xi) \sin \frac{n \pi \xi}{a} d \xi \\
& =\sin \frac{n \pi \xi}{a}[\operatorname{ch} \kappa, \kappa \operatorname{sh} \kappa, \operatorname{sh} \kappa, \kappa \operatorname{ch} \kappa] \operatorname{col}\left(-\lambda+\operatorname{th} \lambda+\lambda \operatorname{th}^{2} \lambda,-\operatorname{th} \lambda,-1,1\right) .
\end{aligned}
$$

To determine the function $q(\xi)$ we put

$$
q(\xi)=\sum_{n=1}^{\infty} c_{n} \sin \frac{n \pi \xi}{a},
$$

and then

$$
\int_{0}^{a} q(\xi) \sin \frac{n \pi \xi}{a} d \xi=c_{n} \int_{0}^{a} \sin ^{2} \frac{n \pi \xi}{a} d \xi=\frac{c_{n} a}{2} .
$$

By substitution $c_{n}$ is found to be

$$
\begin{aligned}
c_{n}=\frac{2}{a} \sin \frac{n \pi \xi}{a} & \frac{1}{\lambda-\operatorname{th} \lambda-\lambda \operatorname{th}^{2} \lambda}[\operatorname{ch} \kappa, \kappa \operatorname{sh} \kappa, \operatorname{sh} \kappa, \kappa \operatorname{ch} \kappa] \\
& \times \operatorname{col}\left(-\lambda+\operatorname{th} \lambda+\lambda \operatorname{th}^{2} \lambda,-\operatorname{th} \lambda,-1,1\right),
\end{aligned}
$$


in virtue of which $w_{\mathrm{II}}^{\prime}$ becomes

$$
\begin{aligned}
w_{\mathrm{II}}^{\prime}=\frac{P a^{2}}{2 \pi^{3} D} \sum_{n=1}^{\infty} \frac{c_{n}}{n^{3}}[\operatorname{ch} \rho, \rho \operatorname{sh} \rho, \operatorname{sh} \rho, \rho \operatorname{ch} \rho] \\
\quad \times \operatorname{col}\left(-\lambda+\operatorname{th} \lambda+\lambda \operatorname{th}^{2} \lambda,-\operatorname{th} \lambda,-1,1\right) \sin \frac{n \pi x}{a} .
\end{aligned}
$$

Thus the wanted deflection $w$ in the domain $0<y<\eta$ is given by the sum

$$
w=\left[w_{\mathrm{II}}^{\prime} \text { of eq. (38) }\right]+\left[w_{\mathrm{I}} \text { of eq. (33) }\right] \text {. }
$$

Similar consideration will enable us to obtain deflections for the remaining domains $-\frac{1}{2} b<y<0$ and $\eta<y<\frac{1}{2} b$. Furthermore, the three-spanned continuous plate may be treated similarly.

The second example is the case of circular plate subjected to an eccentric concentrated load, whose outer edge is clamped or simply supported (Fig. 11). The differential equation for deflection $w$, instead of equation (15), is

$$
\nabla^{4} w=\left(\frac{\partial^{2}}{\partial \rho^{2}}+\frac{1}{\rho} \frac{\partial}{\partial \rho}+\frac{1}{\rho^{2}} \frac{\partial^{2}}{\partial \theta^{2}}\right)^{2} w=0
$$

and solutions suitable for the present example are

$$
\begin{aligned}
w=R_{0}(\kappa, \rho)+ & R_{1}(\kappa, \rho) \cos \theta \\
& +\sum_{n=2}^{\infty} R_{n}(\kappa, \rho) \cos n \theta,
\end{aligned}
$$

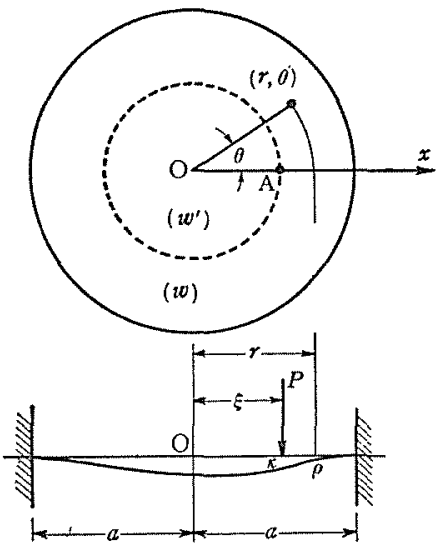

Fig. 11.

where

$$
\left.\begin{array}{l}
R_{0}(\kappa, \rho)=A_{0}(\kappa)+B_{0}(\kappa) \rho^{2}+C_{0}(\kappa) \log \rho+D_{0}(\kappa) \rho^{2} \log \rho, \\
R_{1}(\kappa, \rho)=A_{1}(\kappa) \rho+B_{1}(\kappa) \rho^{3}+C_{1}(\kappa) \rho^{-1}+D_{1}(\kappa) \rho \log \rho, \\
R_{n}(\kappa, \rho)=A_{n}(\kappa) \rho^{n}+B_{n}(\kappa) \rho^{n+2}+C_{n}(\kappa) \rho^{-n}+D_{n}(\kappa) \rho^{-n+2}
\end{array}\right\}
$$

$\kappa, \rho$ representing the dimensionless quantities

$$
\kappa=\frac{\xi}{a}, \quad \rho=\frac{r}{a} .
$$

Let $w$ be denoted by deflection for the domain $\kappa<\rho<1$, and then deflection $w^{\prime}$ for the domain $0<\rho<\kappa$ is given by interchanging $\rho$ and $\kappa$ in equation (40) in virtue of the reciprocal theorem. Hence 


$$
w^{\prime}=R_{0}(\rho, \kappa)+R_{1}(\rho, \kappa) \cos \theta+\sum_{n=2}^{\infty} R_{n}(\rho, \kappa) \cos n \theta .
$$

Comparing equation (40) with equation (42), we must have

$$
\left(\begin{array}{l}
A_{0}(\kappa) \\
B_{0}(\kappa) \\
C_{0}(\kappa) \\
D_{0}(\kappa)
\end{array}\right]=\left(\begin{array}{ll}
a_{1} & a_{2} \\
b_{1} & b_{2} \\
c_{1} & c_{2} \\
d_{1} & d_{2}
\end{array}\right]\left(\begin{array}{l}
1 \\
\kappa^{2}
\end{array}\right),\left(\begin{array}{l}
A_{1}(\kappa) \\
B_{1}(\kappa) \\
C_{1}(\kappa) \\
D_{1}(\kappa)
\end{array}\right)=\left(\begin{array}{ll}
a_{1}^{\prime} & a_{2}^{\prime} \\
b_{1}^{\prime} & b_{2}^{\prime} \\
c_{1}^{\prime} & c_{2}{ }^{\prime} \\
d_{1}^{\prime} & d_{2}^{\prime}
\end{array}\right)\left(\begin{array}{l}
\kappa \\
\kappa^{3}
\end{array}\right),\left(\begin{array}{l}
A_{n}(\kappa) \\
B_{n}(\kappa) \\
C_{n}(\kappa) \\
D_{n}(\kappa)
\end{array}\right)=\left(\begin{array}{ll}
a_{1}^{\prime \prime} & a_{2}^{\prime \prime} \\
b_{1}^{\prime \prime} & b_{2}^{\prime \prime} \\
c_{1}^{\prime \prime} & c_{2}^{\prime \prime} \\
d_{1}^{\prime \prime} & d_{2}^{\prime \prime}
\end{array}\right)\left(\begin{array}{l}
\kappa^{n} \\
\kappa^{n+2}
\end{array}\right),(43)
$$

where $a_{1}, a_{2}, \cdots, d_{2}^{\prime \prime}$ do not depend on $\kappa$. It is noted here that terms which become infinite as $\rho$ tends to zero are excluded, since there is no hole at the center of the plate. Thus equations (40) and (42) take the forms

$$
\begin{gathered}
w=\left[1, \rho^{2}, \log \rho, \rho^{2} \log \rho\right]\left[\begin{array}{ll}
a_{1} & a_{2} \\
b_{1} & b_{2} \\
c_{1} & c_{2} \\
d_{1} & d_{2}
\end{array}\right]\left(\begin{array}{l}
1 \\
\kappa^{2}
\end{array}\right]+\left[\rho, \rho^{3}, \rho^{-1}, \rho \log \rho\right]\left(\begin{array}{ll}
a_{1}^{\prime} & a_{2}^{\prime} \\
b_{1}^{\prime} & b_{2}^{\prime} \\
c_{1}^{\prime} & c_{2}^{\prime} \\
d_{1}^{\prime} & d_{2}^{\prime}
\end{array}\right]\left(\begin{array}{l}
\kappa \\
\kappa^{3}
\end{array}\right) \cos \theta \\
+\sum_{n=2}^{\infty}\left[\rho^{n}, \rho^{n+2}, \rho^{-n}, \rho^{-n+2}\right]\left[\begin{array}{ll}
a_{1}^{\prime \prime} & a_{2}^{\prime \prime} \\
b_{1}^{\prime \prime} & b_{2}{ }^{\prime \prime} \\
c_{1}^{\prime \prime} & c_{2}{ }^{\prime \prime} \\
d_{1}^{\prime \prime} & d_{2}^{\prime \prime}
\end{array}\right]\left[\begin{array}{l}
\kappa^{n} \\
\kappa^{n+2}
\end{array}\right) \cos n \theta
\end{gathered}
$$

$$
\begin{aligned}
& w^{\prime}=\left[1, \rho^{2}\right]\left[\begin{array}{llll}
a_{1} & b_{1} & c_{1} & d_{1} \\
a_{2} & b_{2} & c_{2} & d_{2}
\end{array}\right]\left(\begin{array}{c}
1 \\
\kappa^{2} \\
\log \kappa \\
\kappa^{2} \log \kappa
\end{array}\right)+\left[\rho, \rho^{3}\right]\left[\begin{array}{llll}
a_{1}{ }^{\prime} & b_{1}{ }^{\prime} & c_{1}{ }^{\prime} & d_{1}{ }^{\prime} \\
a_{2}{ }^{\prime} & b_{2}{ }^{\prime} & c_{2}{ }^{\prime} & d_{2}{ }^{\prime}
\end{array}\right)\left(\begin{array}{c}
\kappa \\
\kappa^{3} \\
\kappa^{-1} \\
\kappa \log \kappa
\end{array}\right) \cos \theta \\
& +\sum_{n=2}^{\infty}\left[\rho^{n}, \rho^{n+2}\right]\left(\begin{array}{llll}
a_{1} \prime & b_{1}^{\prime \prime} & c_{1}^{\prime \prime} & d_{1}^{\prime \prime} \\
a_{2}^{\prime \prime} & b_{2}^{\prime \prime} & c_{2}^{\prime \prime} & d_{2}^{\prime \prime}
\end{array}\right)\left(\begin{array}{c}
\kappa^{n} \\
\kappa^{n+2} \\
\kappa^{-n} \\
\kappa^{-n+2}
\end{array}\right) \cos n \theta
\end{aligned}
$$


The continuity conditions along the circle $\rho=\kappa$ are

$$
\left.\begin{array}{l}
\left(\frac{\partial w}{\partial \rho}\right)_{\rho=\kappa}=\left(\frac{\partial w^{\prime}}{\partial \rho}\right)_{\rho=\kappa}, \quad\left(\frac{\partial^{2} w}{\partial \rho^{2}}\right)_{\rho=\kappa}=\left(\frac{\partial^{2} w^{\prime}}{\partial \rho^{2}}\right)_{\rho=\kappa}, \\
\left(\frac{\partial^{3} w}{\partial \rho^{3}}\right)_{\rho=\kappa}-\left(\frac{\partial^{3} w^{\prime}}{\partial \rho^{3}}\right)_{\rho=\kappa}=\frac{P a^{2}}{\pi \kappa D}\left(\frac{1}{2}+\sum_{n=1}^{\infty} \cos n \theta\right)
\end{array}\right\},
$$

in which the right-hand side of the last equation is the expression that the concentrated load $P$ is written in terms of Fourier cosine series along the circle. We shall treat the continuity conditions (46), and after that boundary conditions along the outer edge $(\rho=1)$ will be considered.

We now have the successive derivatives

$$
\begin{aligned}
& \left(\begin{array}{c}
1 \\
\frac{\partial}{\partial \rho} \\
\frac{\partial^{2}}{\partial \rho^{2}} \\
\frac{\partial^{3}}{\partial \rho^{3}}
\end{array}\right)\left[\begin{array}{llll}
e_{1} & e_{2} & e_{3} & e_{4}
\end{array}\right]\left(\begin{array}{c}
1 \\
\rho^{2} \\
\log \rho \\
\rho^{2} \log \rho
\end{array}\right)=\left(\begin{array}{cccc}
e_{1} & e_{2} & e_{3} & e_{4} \\
0 & 2 e_{2}+e_{4} & e_{3} & 2 e_{4} \\
0 & 2 e_{2}+3 e_{4} & -e_{3} & 2 e_{4} \\
0 & 0 & 2 e_{3} & 2 e_{4}
\end{array}\right)\left(\begin{array}{cccc}
1 & 0 & 0 & 0 \\
\rho^{2} & \rho & 1 & 0 \\
\log \rho & \rho^{-1} & \rho^{-2} & \rho^{-3} \\
\rho^{2} \log \rho & \rho \log \rho \log \rho & \rho^{-1}
\end{array}\right), \\
& \left(\begin{array}{c}
1 \\
\frac{\partial}{\partial \rho} \\
\frac{\partial^{2}}{\partial \rho^{2}} \\
\frac{\partial^{3}}{\partial \rho^{3}}
\end{array}\right)\left[\begin{array}{cccc}
e_{1} & e_{2} & e_{3} & e_{4}
\end{array}\right]\left(\begin{array}{c}
\rho \\
\rho^{3} \\
\rho^{-1} \\
\rho \log \rho
\end{array}\right)=\left(\begin{array}{cccc}
e_{1} & e_{2} & e_{3} & e_{4} \\
e_{1}+e_{4} & 3 e_{2} & -e_{3} & e_{4} \\
0 & 6 e_{2} & 2 e_{3} & e_{4} \\
0 & 6 e_{2} & -6 e_{3} & -e_{4}
\end{array}\right)\left(\begin{array}{cccc}
\rho & 1 & \jmath & 0 \\
\rho^{3} & \rho^{2} & \rho & 1 \\
\rho^{-1} & \rho^{-2} & \rho^{-3} & \rho^{-4} \\
\rho \log \rho & \log \rho & \rho^{-1} & \rho^{-2}
\end{array}\right), \\
& \left(\begin{array}{c}
\frac{\partial}{\partial} \\
\frac{\partial}{\partial \rho} \\
\frac{\partial^{2}}{\partial \rho^{2}} \\
\frac{\partial^{3}}{\partial \rho^{3}}
\end{array}\right)\left[\begin{array}{llll}
e_{1} & e_{2} & e_{3} & e_{4}
\end{array}\right]\left(\begin{array}{c}
\rho^{n} \\
\rho^{n+2} \\
\rho^{-n} \\
\rho^{-n+2}
\end{array}\right) \\
& =\left(\begin{array}{cccc}
e_{1} & e_{2} & e_{3} & e_{4} \\
n e_{1} & (n+2) e_{2} & -n e_{3} & -(n-2) e_{4} \\
n(n-1) e_{1} & (n+1)(n+2) e_{2} & n(n+1) e_{3} & (n-1)(n-2) e_{4} \\
n(n-1)(n-2) e_{1} & n(n+1)(n+2) e_{2} & -n(n+1)(n+2) e_{3} & -n(n-1)(n-2) e_{4}
\end{array}\right)
\end{aligned}
$$




$$
\times\left[\begin{array}{llll}
\rho^{n} & \rho^{n-1} & \rho^{n-2} & \rho^{n-3} \\
\rho^{n+2} & \rho^{n+1} & \rho^{n} & \rho^{n-1} \\
\rho^{-n} & \rho^{-n-1} & \rho^{-n-2} & \rho^{-n-3} \\
\rho^{-n+2} & \rho^{-n+1} & \rho^{-n} & \rho^{-n-1}
\end{array}\right],
$$

where $e$ represents one of $a, b, \cdots, d^{\prime \prime}$, and the products of matrices should be taken to consist of diagonal elements alone.

Referring to the successive derivatives above, the continuity conditions (46) afford, with equations (44) and (45), the following:

$$
\left[\begin{array}{ll}
a_{1} & a_{2} \\
b_{1} & b_{2} \\
c_{1} & c_{2} \\
d_{1} & d_{2}
\end{array}\right]=\left[\begin{array}{cc}
a_{1} & a_{2} \\
a_{2}-\alpha & b_{2} \\
0 & \alpha \\
\alpha & 0
\end{array}\right],\left[\begin{array}{ll}
a_{1}^{\prime} & a_{2}^{\prime} \\
b_{1}^{\prime} & b_{2}^{\prime} \\
c_{1}^{\prime} & c_{2}^{\prime} \\
d_{1}^{\prime} & d_{2}^{\prime}
\end{array}\right]=\left[\begin{array}{cc}
a_{1}^{\prime} & a_{2}^{\prime} \\
a_{2}^{\prime} & b_{2}^{\prime} \\
0 & -\frac{\alpha}{2} \\
-2 \alpha & 0
\end{array}\right],\left[\begin{array}{ll}
a_{1}^{\prime \prime} & a_{2}^{\prime \prime} \\
b_{1}^{\prime \prime} & b_{2}^{\prime \prime} \\
c_{1}^{\prime \prime} & c_{2}^{\prime \prime} \\
d_{1}^{\prime \prime} & d_{2}^{\prime \prime}
\end{array}\right]=\left[\begin{array}{cc}
a_{1}^{\prime \prime} & a_{2}^{\prime \prime} \\
a_{2}^{\prime \prime} & b_{2}^{\prime \prime} \\
0 & -\frac{\alpha}{n(n+1)} \\
\frac{\alpha}{n(n-1)} & 0
\end{array}\right],
$$

where for brevity $\alpha=\frac{P a^{2}}{8 \pi D}$.

Equations (47) are the continuity conditions, apart from the boundary conditions along the outer edge $\rho=1$. In respective matrices three constants still remain undetermined, and they will become determinate when the outer boundary conditions are taken into consideration.

We take the outer edge to be of clamped support, which is expressed by the equations

$$
(w)_{\rho=1}=0, \quad\left(\frac{\partial w}{\partial \rho}\right)_{\rho=1}=0 .
$$

Equations (48) give

$$
\left.\begin{array}{cc}
a_{i}+b_{i}=0, & 2 b_{i}+c_{i}+d_{i}=0 \\
a_{i}{ }^{\prime}+b_{i}{ }^{\prime}+c_{i}^{\prime}=0, & a_{i}^{\prime}+3 b_{i}{ }^{\prime}-c_{i}{ }^{\prime}+d_{i}^{\prime}=0 ; \\
a_{i}^{\prime \prime}+b_{i}^{\prime \prime}+c_{i}^{\prime \prime}+d_{i}^{\prime \prime}=0, & n a_{i}^{\prime \prime}+(n+2) b_{i}^{\prime \prime}-n c_{i}^{\prime \prime}-(n-2) d_{i}^{\prime \prime}=0,
\end{array}\right\}
$$

where $n=1,2, \cdots$. Substituting equations (47) into equations (49), the coefficient matrices become determinate.

Thus the wanted deflections $w$ (for $\kappa<p<1$ ) and $w^{\prime}$ (for $0<p<\kappa$ ) become respectively 


$$
\begin{aligned}
& w=\alpha\left[1, \rho^{2}, \log \rho, \rho^{2} \log \rho\right]\left[\begin{array}{cc}
1 & \frac{1}{2} \\
-1 & -\frac{1}{4} \\
0 & 1 \\
1 & 0
\end{array}\right]\left[\begin{array}{l}
1 \\
\kappa^{2}
\end{array}\right]+\alpha\left[\rho, \rho^{3}, \rho^{-1}, \rho \log \rho\right]\left[\begin{array}{cc}
-1 & 1 \\
1 & -\frac{3}{2} \\
0 & -\frac{3}{3} \\
-2 & 0
\end{array}\right]\left[\begin{array}{l}
n \\
\kappa^{3}
\end{array}\right] \cos \theta \\
& +\sum_{n=2}^{\infty} \alpha\left[\rho^{n}, \rho^{n+2}, \rho^{-n}, \rho^{-n+2}\right]\left(\begin{array}{cc}
-\frac{1}{n-1} & \frac{1}{n} \\
\frac{1}{n} & -\frac{1}{n+1} \\
0 & -\frac{1}{n(n+1)} \\
\frac{1}{n(n-1)} & 0
\end{array}\right)\left[\begin{array}{c}
\kappa^{n} \\
\kappa^{n+2}
\end{array}\right] \cos n \theta \\
& w^{\prime}=\alpha\left[1, \rho^{2}\right]\left[\begin{array}{cccc}
1 & -\frac{1}{2} & 0 & 1 \\
\frac{1}{2} & -\frac{1}{2} & 1 & 0
\end{array}\right]\left[\begin{array}{c}
1 \\
\kappa^{2} \\
\log \kappa \\
\kappa^{2} \log \kappa
\end{array}\right]+\alpha\left[\rho, \rho^{3}\right]\left(\begin{array}{cccc}
-1 & 1 & 0 & -2 \\
1 & -\frac{1}{2} & -\frac{1}{2} & 0
\end{array}\right)\left[\begin{array}{c}
\kappa \\
\kappa^{3} \\
\kappa^{-1} \\
\kappa \log \kappa
\end{array}\right] \cos \theta \\
& +\sum_{n=2}^{\infty} \alpha\left[\rho^{n}, \rho^{n+2}\right]\left(\begin{array}{cccc}
-\frac{1}{n-1} & \frac{1}{n} & 0 & \frac{1}{n(n-1)} \\
\frac{1}{n}-\frac{1}{n+1} & -\frac{1}{n(n+1)} & 0
\end{array}\right)\left(\begin{array}{c}
\kappa^{n} \\
\kappa^{n+2} \\
\kappa^{-n} \\
n^{-n+2}
\end{array}\right) \cos n \theta,
\end{aligned}
$$

where as before

$$
\alpha=\frac{P a^{2}}{8 \pi D}, \quad \kappa=\frac{\xi}{a}, \quad \rho=\frac{r}{a} .
$$

The usual forms of expression for equtions (50) and (51) are not so complicated, and they take the forms

$$
\begin{aligned}
w= & \frac{P a^{2}}{8 \pi D}\left[\left(\rho^{2}+\kappa^{2}\right) \log \rho+\frac{1}{2}\left(1+\kappa^{2}\right)\left(1-\rho^{2}\right)\right] \\
& -\frac{P a^{2}}{8 \pi D} \kappa\left[\left(1-\kappa^{2}\right) \rho-\frac{1}{4}\left(2-\kappa^{2}\right) \rho^{3}+\frac{1}{2} \kappa^{2} \rho^{-1}+2 \rho \log \rho\right] \cos \theta \\
& +\frac{P a^{2}}{8 \pi D} \sum_{n=2}^{\infty} \kappa^{n}\left[-\left(\frac{1}{n-1}-\frac{\kappa^{2}}{n}\right) \rho^{n}+\left(\frac{1}{n}-\frac{\kappa^{2}}{n+1}\right) \rho^{n+2}\right.
\end{aligned}
$$




$$
\begin{gathered}
\left.-\frac{\kappa^{2}}{n(n+1)} \rho^{-n}+\frac{1}{n(n-1)} \rho^{-n+2}\right] \cos n \theta \quad(\kappa<\rho<1), \\
w^{\prime}=\frac{P a^{2}}{8 \pi D}\left[\left(\kappa^{2}+\rho^{2}\right) \log \kappa+1\left(1+\rho^{2}\right)\left(1-\kappa^{2}\right)\right] \\
-\frac{P a^{2}}{8 \pi D} \rho\left[\left(1-\rho^{2}\right) \kappa-\frac{1}{2}\left(2-\rho^{2}\right) \kappa^{3}+\frac{1}{2} \rho^{2} \kappa^{-1}+2 \kappa \log \kappa\right] \cos \theta \\
+\frac{P a^{2}}{8 \pi D} \sum_{n=2}^{\infty} \rho^{n}\left[-\left(\frac{1}{n-1}-\frac{\rho^{2}}{n}\right) \kappa^{n}+\left(\frac{1}{n}-\frac{\rho^{2}}{n+1}\right) \kappa^{n+2}\right. \\
\left.-\frac{\rho^{2}}{n(n+1)} \kappa^{-n}+\frac{1}{n(n-1)} \kappa^{-n+2}\right] \cos n \theta \quad(0<\rho<\kappa) .
\end{gathered}
$$

We see that the above result is the same as that obtained by the ordinary procedure.

$$
\text { If, instead of equations (48), we take }
$$

$$
(w)_{\rho=1}=0, \quad\left[\frac{\partial^{2} w}{\partial \rho^{2}}+\nu\left(\frac{1}{\rho} \frac{\partial w}{\partial \rho}+\frac{1}{\rho^{2}} \frac{\partial^{2} w}{\partial \theta^{2}}\right)\right]_{\rho=1}=0
$$

we then have the case in which the outer edge of the circular plate is simply supported; $\nu$ being Poisson's ratio of the plate. The rest of this case is here omitted.

In conclusion, it is added that the present report is confined to the cases whose solutions are known otherwise. But several additional examples, which are more complicated and seem their solutions not to have been given owing to the complexity, have been worked out, and they will appear some other day.

Acknowledgement. I should like to express my sincere thanks to Dr. Y. TANAKA, Professor Emeritus of Civil Engineering, University of Tokyo, and Member of Japan Academy, for his constant encouragements. 


\section{References}

1) For instance, R. J. RoARK, "Formulas for Stress and Strain", 1st ed. (1938), p. 92.

2) Loc. cit., p. 94 .

3) Loc. cit., p. 97.

4) Loc. cit., p. 100.

5) S. Timoshenko and WoInowsky-KRIEGER, "Theory of Plates and Shells;" 2nd ed. (1959), p. 290. 